\title{
Ultrasonic NDE for internal defect detection in multi-layered composite materials by multi-resolution signal decomposition
}

\begin{abstract}
Ultrasonic NDE has been a well known approach to investigate material's microstructures, mechanical properties and structure integrity in industry. The internal structure of a material and position of anomalies can be recognised by the reactions of different materials to ultrasound. However, the interpretation of ultrasound signals is difficult in composite material inspection task due to the fact that the ultrasonic pulse is reflected not only by the defect occurred within the material but the microstructures and multiple lay ups of the material. This phenomenon causes the backscattering noise to hinder the real defect's signal during the inspection. Backscattering noise exists in multiple frequencies. The objective of this study was to develop a new noise reduction method to enhance the defect detectability in coarse-grained structure material such as composites materials. This method increases Signalto-noise Ratio (SNR) by means of decomposing the original signal into multiresolution representations. To prevent the loss of information, the signal is processed in both temporal and frequency domain. The proposed method has been tested on simulated signal and Glass Fiber Reinforced Plastics (GFRP) laminates. Both simulation and experimental results showed that this method can significantly reduce grain noise while preserving the resolution of the original signal of the defect.
\end{abstract}

Keyword: Ultrasound; Signal-to-noise ratio; Wavelets; Composite materials; Glass fiber reinforced plastics 